\title{
Resistant obsessive compulsive disorder (ROC): predictive factors and links with soft bipolarity Elie-Georges Hantouche*1, Christophe Demonfaucon ${ }^{2}$ and Daniel Gerard 3
}

\author{
Address: ${ }^{1}$ Pitié-Salpetriere Hospital, Paris, France, ${ }^{2}$ AFTOC, Chateaufort, France and ${ }^{3}$ Sanofi-Aventis, France \\ * Corresponding author
}

\author{
from International Society on Brain and Behaviour: 2nd International Congress on Brain and Behaviour \\ Thessaloniki, Greece. 17-20 November 2005 \\ Published: 28 February 2006 \\ Annals of General Psychiatry 2006, 5(SuppI I):SI4I doi:I0.I I86/I744-859X-5-SI-SI4I
}

\section{Background}

In the collaborative survey of AFTOC (French Association of patients suffering from OCD) in which 612 OCD patients were included, we observed a high rate of soft bipolar comorbidity in OCD: $30 \%$ with hypomanic episodes and 50\% with cyclothymia, "Cyclothymic OCD" appeared as a distinct form with a different clinical picture, episodic course of illness; higher rate of recurrent depression; suicide attempts and psychiatric admissions; and less favorable response to anti-OCD drug therapy (Hantouche et al., 2003).

\section{Materials and methods}

Following these data, a new survey of AFTOC was implemented with the aim of exploring ROC, Resistant OCD.

\section{Results}

The new survey of AFTOC "TOC and ROC" have selected a sample of 360 patients, who are members of the association. The rate of ROC was $44.2 \%, 25.3 \%$ of Good responders (GR), and $30.5 \%$ in between. Inter-group comparisons (vs GR) showed in the ROC group significant higher rates of psychiatric admissions (49\% vs $28 \%$ ), suicide attempts ( $26 \%$ vs $13 \%)$, cases with slow and continuous course of illness ( $69 \%$ vs $48 \%, \mathrm{p}=0.001$ ); higher numbers of doctors consulted (5.5 vs 3.2), compulsions (4.6 vs 3.4 ), and psychiatric comorbidity ( 2.8 vs 2.0 , especially agoraphobia, depression and worry about appearance). Rate of worsening under SRI drugs was higher in ROC $(21 \%$ vs $10 \%(\mathrm{p}=0.0115)$, and also of mood switching $(42 \%$ vs $27 \%$, p < 0.001$)$. Cyclothymic Temp ( $63 \%$ vs $43 \%, \mathrm{p}=0.003)$, depressive Temp $(72 \%$ vs $53 \%, \mathrm{p}=$ $0.004)$, and Irritable Temp ( $21 \%$ vs $9 \%, p=0.02)$ were more represented in ROC. Logistic regression analyses (Stepwise method) showed that the most powerful factors were slow and continuous course of illness $(\mathrm{OR}=2.2)$, worsening under SRIs $(\mathrm{OR}=2.9)$ age above 40 yrs $(\mathrm{OR}=$ $2.7)$, psychiatric admission $(\mathrm{OR}=2.2)$, and worry about appearance $(\mathrm{OR}=2.8)$.

\section{Discussion}

We submit the hypothesis that cases with ROC should be explored through specific comorbidity (obsession of appearance, and Cyclothymic depression), and especially worsening with serotonergic antidepressants. More vigilance is needed toward suicide risk in this condition.

\section{References}

I. Hantouche EG, et al:: Cyclothymic OCD: a distinct entity? J Affect Disord 2003, 75: I- 10 . 